\title{
Stress Problem Identified
}

National Cancer Institute

\section{Source}

National Cancer Institute. Stress Problem Identified. NCI Thesaurus. Code C92120.

Problems caused by either excessive or inadequate physical force exerted on it by another object resulting in problems e.g. wear, bending, deformation, fracture, fatigue. 